\title{
La escala Campbell para la valoración del dolor en urgencias/emergencias. El caso de Turkana, Kenya
}

\author{
Elena García Higón' ', Andrés Climent Rubio², José Ramón Martínez Riera³ \\ `Enfermera, cooperante en Turkana (Kenya). Coordinadora del Grupo de Trabajo de Cooperación Internacional y \\ Transculturalidad de la Asociación de Enfermería Comunitaria (AEC) \\ ¿2Enfermero, cooperante en Turkana (Kenya). Vocal del Grupo de Trabajo de Cooperación Internacional y \\ Transculturalidad de la Asociación de Enfermería Comunitaria (AEC) \\ ${ }^{3}$ Doctor por la Universidad de Alicante. Facultad de Ciencias de la Salud. Universidad de Alicante. Presidente de la \\ Asociación de Enfermería Comunitaria (AEC)
}

Fecha de recepción: 8 de junio de 2016. Aceptada su publicación: 10 de enero de 2017.

\section{Resumen}

Objetivo: estudiar la viabilidad de una escala de valoración del dolor que contemple rasgos conductuales que ofrezcan al persona sanitario una valoración no solo basada en la interpretación del paciente de su dolor en la zona rural de Turkana (Kenya).

Método: se realizó un estudio descriptivo observacional. Se seleccionaron personas de entre 18 y 65 años pertenecientes a la cultura turkana con dolor en situaciones de urgencia, y se dividieron en dos grupos. En el Grupo 1 se aplican la escala visual analógica (EVA) y la escala con caras (FPS), y en el Grupo 2 la escala CampbeII. Mediante el índice Spearman se analizó la correlación entre las tres escalas.

Resultados: se estudiaron 48 adultos, 32 en el Grupo 1 y 16 en el Grupo 2. Mientras las escalas EVA y FPS mantienen una buena correlación de 0,667 entre ellas, la escala Campbell con cada una de ellas ofrece unos valores de 0,245 y 0,36, respectivamente, por tanto, no parece existir relación entre esta con cada una de las otras

Conclusiones: la valoración con escalas basadas en la asociación de un número o una cara muestran deficiencias, mientras que e resultado de un uso combinado de estas con una escala como la escala Campbell es más satisfactorio.

\section{Palabras clave}

Dolor; diferencias culturales; escalas valoración; urgencias; Campbell; investigación.

\section{Campbell Scale for pain assessment in Hospital Emergency Units. The case of Turkana, Kenya}

Objective: to study the feasibility of a pain assessment scale that considers behavioural features and that can provide the healthcare staff with an assessment not only based on the interpretation of their pain by patients, in the rural area of Turkana. Method: a descriptive observational study was conducted. People between 18 and 65-year-old were selected, from the Turkana culture, with pain in emergency situations, and they were divided into two groups. For Group 1, the Visual Analogue Scale (VAS) and the Faces Pain Scale (FPS) were used, while the Campbell Scale was used for Group 2. The correlation between the three scales was analyzed through the Spearman Index.

Results: the study included 48 adult patients: 32 in Group 1 and 16 in Group 2. While the VAS and FPS maintained a good 0.667 correlation between them, the Campbell Scale offered values of 0.245 and 0.36 respectively, with each of the other scales; therefore, there did not seem to be an association between this scale and each of the other scales.

Conclusions: assessment with scales based on the association with a number or a face will present failures, while the result of a combined use of these scales with a scale such as the Campbell Scale will be more satisfactory.

\section{Key words}

Pain; cultural differences; assessment scales; Emergency; Campbell; research.

\section{Introducción}

La definición del dolor ha variado enormemente a lo largo de la historia. Aunque de forma popular está visto como algo negativo, es de hecho un proceso esencial que alerta y protege de un mayor perjuicio. Según el Diccionario de la Real Academia de la Lengua, dolor es una "sensación molesta y aflictiva de una parte del cuerpo por causa interior o exterior, o un sentimiento de pena y congoja" (1). La definición que McCaffery dio al dolor en 1968 hace hincapié en que es el paciente, no el proveedor de cuidados de la salud,

quien tiene la autoridad sobre su dolor, cuya propia mención es el indicador más fiable de este (2). Por su parte, la Sociedad Española del Dolor recalca la importancia del dolor para aquel que lo padece, y lo considera más que como un síntoma como una enfermedad (3).

Actualmente, los pacientes se califican siguiendo unas escalas de dolor basadas en su experiencia subjetiva. Esto se ve influenciado por valores culturales y étnicos, creencias religiosas, presiones sociales y estado socioeconómico, lo

\section{Dirección de contacto:}

Elena García Higón. Paseo Alameda, 60-8² 2ª 46023 Valencia. Email: egarciahigon@gmail.com

\section{Cómo citar este artículo:}

García Higón E, Climent Rubio A, Martínez Riera JR. La escala Campbell para la valoración del dolor en urgencias/emergencias. El caso de Turkana, Kenya. Metas Enferm mar 2017; 20(2): 12-17. 
que complica enormemente la valoración (4), haciendo que el dolor se pueda menospreciar o magnificar por parte del personal sanitario. En el Cuadro 1 se comparan las características de algunas de las escalas existentes para la valoración del dolor, así como su graduación, la población en la que se usa cada una de ellas y sus limitaciones.

Los pacientes pertenecientes a diferentes grupos étnicos que padecen dolor necesitan estar capacitados para informar con precisión sobre los niveles de intensidad del mismo, y el personal sanitario que trata a estos pacientes debe desarrollar estrategias específicas para enfrentar los estereotipos negativos que se tienen sobre estos grupos (5). Nortje y Albertyn (6) concluyeron, en un estudio realizado en Sudáfrica, que los proveedores de salud deben ser más conscientes y sensibles a la expresión cultural del dolor, y tener en cuenta que las diferencias en la comunicación del dolor podría influir en su plan de atención.

En África subsahariana, la prevalencia del dolor se ve amplificada por la falta de acceso a servicios de salud, un inadecuado diagnóstico, la falta de educación en referencia al control del dolor y a la escasez de medicamentos (7).

Específicamente en Kenya, existe una gran brecha entre la asistencia y un control satisfactorio del dolor. Kristen Huang et al. (8) adaptaron y tradujeron a la lengua swahili la escala de dolor con caras y la escala numérica verbal, y se demostró su validez aparente y la aceptabilidad a través de una entrevista con pacientes de un hospital de referencia en Kenya. Sin embargo, el personal sanitario en estas áreas no está debidamente entrenado para evaluar el dolor, la valoración del dolor no es habitualmente practicada, y el dolor no es a menudo tratado correctamente debido a la escasa práctica en el uso de analgésicos mayores y la falta de priorización en su alivio (9).

Este estudio se centró en la población adulta de Turkana, al noroeste de Kenya, donde abunda la forma de vida nómada, el acceso a centros sanitarios se ve complicada o por la escasez de estos y por la ausencia o el mal estado de las carreteras, que dificultan la accesibilidad. Añadido a la escasez de recursos, se encuentra una cultura donde la hambruna y las guerrillas tribales con los poblados vecinos de Etiopía y Sudán del Sur han engendrado un concepto firme del dolor y la enfermedad, por los cuales adoptan unas estrategias de afrontamiento y expresión del dolor que dificultan excesivamente la valoración.

Dentro del campo de las urgencias, se observa que es necesaria la validación de una escala que permitiese una pronta evaluación del paciente adulto de estas características para su atención, dadas las dificultades de valoración del dolor que presenta este tipo de comunidades.

Tras rastrear una escala que no se centrara solo en lo referido por el paciente, sino que incluyera también una serie de rasgos conductuales que aportaran al personal sanitario una valoración más objetiva, se propuso la aplicación de la escala de Campbell (Behavioral Pain Assessment Scale) (10) (Cuadro 2), la cual está diseñada no solo para valorar la presencia de dolor, sino para cuantificar su intensidad.

En 2008, la Sociedad Española de Medicina Intensiva y Unidades Coronarias publicó unas recomendaciones de analgesia y sedación en las que se menciona la importancia de la monitorización y la cuantificación del dolor en pacientes críticos sin capacidad de comunicación, por lo que proponían el uso y la validación de la escala Campbell (10).

Los ítems conductuales que contempla esta escala se centran en reacciones generalmente involuntarias comunes en los seres humanos, por tanto, podrían facilitar la evaluación del dolor en los turkana, dado que no se requiere que el paciente tenga la capacidad de relacionar cualquiera de los elementos usados en escalas como la escala visual analógica (EVA) o la Faces Pain Scale (FPS). Sin embargo, el uso de esta escala está poco extendido y necesita ser validada (10).

\begin{tabular}{|c|c|c|c|c|}
\hline Escala & Características & Usada en & Graduación & Limitaciones \\
\hline EVA & $\begin{array}{l}\text { Línea de } 10 \text { cm con o sin } \\
\text { marcas por cada cm }\end{array}$ & $\begin{array}{l}\text { Adultos y pediátricos } \\
\qquad(>4 \text { años })\end{array}$ & $\begin{array}{c}\text { De } 0 \text { (no dolor) a } 10 \text { (peor } \\
\text { dolor imaginable) }\end{array}$ & $\begin{array}{l}\text { Poco precisa en demencia } \\
\text { avanzada o pacientes con } \\
\text { problemas de comunicación } \\
\text { (incapacidad o sedación) }\end{array}$ \\
\hline Escala con caras & $\begin{array}{l}\text { Serie horizontal de seis } \\
\text { rostros }\end{array}$ & Adultos, niños, geriátricos & $\begin{array}{c}\text { De } 0 \text { (expresión facial } \\
\text { neutra, no dolor) a } 10 \\
\text { (expresión facial mayor dolor } \\
\text { posible) }\end{array}$ & $\begin{array}{l}\text { Poco útil en demencia } \\
\text { avanzada o pacientes con } \\
\text { problemas de comunicación } \\
\text { (incapacidad o sedación) }\end{array}$ \\
\hline BPS & $\begin{array}{c}\text { Valora del } 1 \text { al } 4 \text { la expresión } \\
\text { facial, la movilidad y la lucha } \\
\text { o no contra el ventilador }\end{array}$ & $\begin{array}{c}\text { Pacientes críticos, } \\
\text { inconscientes, sedados, } \\
\text { unidades de cuidados críticos }\end{array}$ & $\begin{array}{l}\text { De } 3 \text { (menor dolor) a } 12 \\
\text { (mayor dolor) }\end{array}$ & $\begin{array}{c}\text { Poca utilidad para cuantificar } \\
\text { la intensidad del dolor }\end{array}$ \\
\hline CPOT & $\begin{array}{c}\text { Valora del } 0 \text { a } 2 \text { la expresión } \\
\text { facial, movimiento corporal, } \\
\text { tensión muscular y } \\
\text { asincronía con el ventilador/ } \\
\text { vocalización }\end{array}$ & $\begin{array}{c}\text { Pacientes críticos, } \\
\text { inconscientes, sedados, } \\
\text { unidades de cuidados críticos }\end{array}$ & $\begin{array}{l}\text { De } 0 \text { (menor dolor) a } 8 \\
\text { (mayor dolor) }\end{array}$ & $\begin{array}{c}\text { Califica la intensidad de las } \\
\text { reacciones conductuales, no } \\
\text { del dolor en sí }\end{array}$ \\
\hline
\end{tabular}

Fuente: elaboración propia.

EVA: escala visual analógica; BPS: Behavioral Pain Scale; CPOT: Critical-Care Pain Observation Tool 


\begin{tabular}{|c|c|}
\hline \multicolumn{2}{|l|}{ Cuadro 2. Escala de Campbell (10) } \\
\hline Musculatura facial & Puntuación \\
\hline $\begin{array}{l}\text { Relajada } \\
\text { En tensión, ceño fruncido y/o mueca de dolor } \\
\text { Ceño fruncido de forma habitual y/o dientes } \\
\text { apretados }\end{array}$ & $\begin{array}{l}0 \\
1 \\
2\end{array}$ \\
\hline Tranquilidad & Puntuación \\
\hline $\begin{array}{l}\text { Tranquilo, relajado, movimientos normales } \\
\text { Movimientos ocasionales de inquietud y/o de posición } \\
\text { Movimientos frecuentes, incluyendo cabeza o } \\
\text { extremidades }\end{array}$ & $\begin{array}{l}0 \\
1 \\
2\end{array}$ \\
\hline Tono muscular & Puntuación \\
\hline $\begin{array}{l}\text { Normal } \\
\text { Aumentado. Flexión de dedos de manos y/o pies } \\
\text { Rígido }\end{array}$ & $\begin{array}{l}0 \\
1 \\
2\end{array}$ \\
\hline Respuesta verbal & Puntuación \\
\hline $\begin{array}{l}\text { Normal } \\
\text { Quejas, lloros, quejidos o gruñidos ocasionales } \\
\text { Quejas, lloros, quejidos o gruñidos frecuentes }\end{array}$ & $\begin{array}{l}0 \\
1 \\
2\end{array}$ \\
\hline Confortabilidad & Puntuación \\
\hline $\begin{array}{l}\text { Confortable y/o tranquilo } \\
\text { Se tranquiliza con el tacto y/o la voz. Fácil de distraer } \\
\text { Difícil de confortar con el tacto o hablándole }\end{array}$ & $\begin{array}{l}0 \\
1 \\
2\end{array}$ \\
\hline
\end{tabular}

Graduación del dolor: 0 no dolor; 1 -3 dolor leve-moderado; 4-6 dolor moderado-grave; > 6 dolor muy intenso. Objetivo: $<3$

La hipótesis del presente trabajo es que la escala Campbell podría ser más válida y fiable para la población turkana, del norte de Kenya, con un mejor manejo del dolor posterior.

El objetivo fue determinar la mejor escala adaptada a la población turkana. La contribución a la validación de la escala Campbell puede además aportar elementos para la generalizabilidad de los resultados a otros países con poblaciones de características similares en un contexto de urgencias.

\section{Método}

Se realizó un estudio descriptivo transversal.

El ámbito de estudio fue la población turkana; esta población asciende a casi 900.000 habitantes, de los cuales alrededor del 60\% mantiene una forma de vida nómada. La muestra fue de 48 adultos (24 hombres y 24 mujeres), que cumplían los siguientes criterios de inclusión: hombres y mujeres en edad adulta (mayores de 14 años y menos de 65), pertenecientes a la cultura turkana, con una forma de vida nómada (no pertenecían a un poblado estable), que presentaran dolor en situación de urgencia con daño tisular visible y cuyo motivo de atención de urgencia fuera caída, herida por arma blanca, herida por arma de fuego, quemadura o picadura de animales (serpiente, escorpión o araña). Fueron excluidas las personas que presentaban incapacidad para hablar y las que tenían dolor crónico.

El equipo investigador comenzó a trabajar en Kenya en enero de 2015, mientras que la realización del estudio empezó en abril en el distrito de Turkana, prolongándose hasta junio. Durante las dos primeras semanas de abril, tres observadores (dos enfermeros españoles y un líder comunitario local capacitado que actuó como mediador social y que, entre otras funciones, se encargaba de traducir e interpretar de forma oral y escrita de inglés a turkana y viceversa) estudiaron las escalas de valoración del dolor, y escogieron la escala Campbell como propuesta para su uso en esta población. Durante la tercera semana, los dos sanitarios se encargaron de explicar su aplicación al mediador social que actuaba como traductor local. A partir de la tercera semana, comenzó la recogida de datos. La selección de los sujetos se realizó consecutivamente a medida que se les atendía, se aplican los criterios de inclusión y exclusión, y aceptaban de forma oral (ya que desconocen la escritura) formar parte del estudio, tras explicarles el objetivo y su participación.

Se dividió la muestra en dos grupos. El Grupo 1 constó de 32 personas, en las cuales se aplicaron, con la ayuda del líder comunitario, las escalas de dolor EVA y FPS, cuya aplicación ya se ha estudiado en África (9) de forma estructurada. Se evaluaron ocho hombres y ocho mujeres con la escala EVA y otros ocho hombres y ocho mujeres con la FPS. Se registró y se analizó de forma aislada sin usar el conocimiento de la cultura para la interpretación. En el Grupo 2, de 16 personas (ocho hombres y ocho mujeres), con la ayuda del mismo líder comunitario, se analizó la situación del dolor con la escala Campbell, mediante observación directa del paciente, y se registró la puntuación obtenida. Esta escala cuenta con cinco ítems conductuales y con un rango de puntuación de 0 (ausencia de dolor) a 10 puntos (máximo dolor). Teniendo en cuenta que su graduación del 0 al 10 la hace más equiparable a las escalas usadas en pacientes conscientes, y que contempla un mayor número de ítems conductuales, lo que podría disminuir el resultado por causas ajenas al dolor, la escala de Campbell podría ser un instrumento adecuado para evaluar la presencia e intensidad del dolor en pacientes críticos y/o no comunicati$\operatorname{vos}(11)$.

El algoritmo seguido para administrar tratamiento farmacológico tras evaluar el dolor con la escala EVA fue el recomendado en la guía de Actuación Enfermera en Urgencias y Emergencias Prehospitalarias del Departamento de Salud de la Generalidad Catalana (12); para la escala FPS se utilizó el mismo que para el de la escala EVA, por su similitud en la valoración del dolor según su puntuación de 0 a 10:

- EVA 1-3 puntos: paracetamol 1 g oral.

- EVA 4-7 puntos: paracetamol+dexketoprofeno 25-50 $\mathrm{mg} I \mathrm{~V}$.

- EVA > 7 puntos: cloruro mórfico 2-3 mg IV (0,05-0,1 $\mathrm{mg} / \mathrm{kg}$ ), repetir en cinco minutos si persiste; o fentanilo $1-3 \mu g / k g$.

Para la escala Campbell no se han encontrado referencias en cuanto a la administración de fármacos según su puntuación. El equipo investigador, de manera consensuada, decidió la siguiente pauta: Campbell 0 (no dolor-dolor muy leve): paracetamol 500 mg oral. 
- Campbell 1-3 puntos (dolor leve-moderado): paracetamol $1 \mathrm{~g} \mathrm{IV}$.

- Campbell 4-6 puntos (dolor moderado-grave): paracetamol 1 g IV+dexketoprofeno $50 \mathrm{mg}$ IV; o diclofenaco 75 $\mathrm{mg} \mid \mathrm{M}$.

- Campbell > 6 puntos (dolor muy intenso): cloruro mórfico 2-3 mg IV (0,05-0,1 mg/kg); o fentanilo 1-3 Ng/kg.

Tras la evaluación con cada escala, se procedió a la administración de fármacos para el tratamiento del dolor según la puntuación obtenida y se reevaluó de nuevo con la escala Campbell en ambos grupos. Los fármacos utilizados fueron analgésicos. No se administraron fármacos hipnóticos como los neurolépticos, las benzodiazepinas o los barbitúricos, ni ansiolíticos o sedantes. Se comparó la diferencia entre la valoración obtenida y la respuesta al tratamiento aplicado.

La muestra fue diferente en cada grupo para aplicar al mismo número de pacientes cada una de las tres escalas, y facilitar así la comparación y la obtención de datos más equitativos. Los sujetos participantes en los tres grupos tienen características similares e intercambiables a efectos de la investigación que se llevó a cabo.

Los tres observadores valoraron el dolor aplicando cada una de las escalas. De la administración del tratamiento, asî como del registro y análisis de los datos y el montaje de las tablas, se encargaron los dos sanitarios. La prescripción y administración del tratamiento se llevó a cabo por estos debido a la ausencia de otro tipo de profesional sanitario en el área.

Respecto a las consideraciones éticas, cabe señalar que las personas pertenecientes a esta población no tienen conocimiento de la existencia o la utilidad de un estudio científico, así como tampoco de un consentimiento informado para poder incluirlos en él. Se le pidió permiso y aprobación al líder comunitario (jefe o chief) para escribir públicamente sobre su pueblo, y a cada uno de los estudiados se les explicó el procedimiento de manera que intentaran entender la utilidad que podrían tener los datos obtenidos sobre ellos para la comunidad científica. Se consideró que el beneficio era mutuo, que podría suponer una útil aportación a la comunidad científica, y, además, una mejora de la calidad de vida de los turkana al evitar el dolor, entendido este como perjudicial dentro de su cultura.

\section{Resultados}

Se estudiaron en total 48 adultos, 24 hombres y 24 mujeres, de la población turkana, con una forma de vida nómada, que presentaban dolor en situación de urgencia con presencia de daño tisular. No se produjo ninguna pérdida.

Mientras que con las escalas EVA y FPS se obtuvieron porcentajes iniciales del 56\% para dolor moderado y del $13 \%$ y $6 \%$ para dolor grave, con la escala Campbell se obtuvo un $25 \%$ de pacientes con dolor moderado y un $69 \%$ con dolor grave. Los porcentajes obtenidos en la evaluación inicial y la reevaluación tras el tratamiento adecuado a la puntuación obtenida en ambos grupos se resumen en la Tabla 1.

La correlación entre escalas se midió con el índice Rho Spearman. Su valor fue de 0,667 entre las escalas EVA y FPS, con una significancia bilateral de 0,005 ( $p<0,01$ ). La correlación entre la escala EVA y la Campbell fue de 0,254, con una significancia bilateral del 0,343. Por otro lado, entre la escala FPS y la Campbell su valor fue 0,36, con una significancia bilateral del 0,171.

\section{Discusión y conclusiones}

Se ha demostrado que la EVA es confiable y válida para muchas poblaciones de pacientes (13), adultos y pediátricos (más útiles en mayores de 4 años) (14), y en diferentes tipos de patologías y estados postoperatorios (15).

En la África subsahariana existe un estudio no muy reciente el cual reveló la correlación que existe entre esta escala y la escala numérica verbal tras aplicarlas para evaluar el dolor en un grupo de 100 pacientes en Nigeria (16). Blum et al. (17) trataron de entender cómo el alfabetismo y las diferencias culturales y lingüísticas afectan a los resultados obtenidos en las escalas de valoración del dolor en 315 pacientes de Uganda y Sudáfrica enfermos de cáncer y VIH. Para ello diseñó la jerrycan scale, una escala en la que se muestra la foto de un recipiente típico en el que se porta agua en estos países, donde "vacío" equivale a "no dolor" y "Ileno" equivale a "el peor dolor imaginable", y la aplicó a este grupo junto con la escala verbal del dolor, la escala con caras y la escala con manos; los resultados mostraron una gran correlación entre las tres últimas, mientras que la escala propuesta resultó tener menor correlación con la escala verbal (17).

\begin{tabular}{l} 
Tabla 1. Porcentajes de puntuación obtenida en la valoración de cada escala, inicialmente y tras reevaluación con Campbell \\
\cline { 2 - 4 } \\
\cline { 2 - 4 }
\end{tabular}


Huang et al. (8) demostraron la validez y la aceptabilidad de la escala FPS en un hospital de Kenya, que junto con la escala numérica verbal fue encontrada fácil de usar por los pacientes. Otro estudio realizado en el mismo país determinó que el 66\% de los pacientes evaluados con las escalas anteriores sufría de dolor aun con tratamiento (18). En estos países, el número de estudios que examinan la validez transcultural de instrumentos de evaluación del dolor es muy escaso $(8,9,16,18)$. Pero estas investigaciones solo demuestran que pacientes de entornos urbanos, sin claras dificultades de expresión del dolor y sin marcadas barreras culturales, entienden y son capaces de relacionar su dolor con un número, pero no tienen en cuenta que estas descripciones pueden estar influidas por su cultura o nivel educativo y no valoran si realmente el número se corresponde realmente al dolor sufrido, obteniendo en algunos casos un dolor deficientemente tratado.

No se han encontrado, además, referencias al uso de otras escalas generalmente empleadas en personas con dificultad en la comunicación, como los pacientes críticos que se hallan bajo algún tipo de sedación en unidades especiales o los pacientes con demencia o problemas psiquiátricos, en este tipo de poblaciones, donde el idioma, la falta de educación o la diferencia cultural suponen una barrera.

En el presente estudio se compara el uso de las escalas EVA y FPS con la escala Campbell. Al igual que en otros trabajos donde se estudia el uso de escalas basadas en la interpretación del paciente de su dolor (13,19), la EVA y la FPS mostraron buena correlación, no así como cada una con la escala Campbell.

Como limitaciones del estudio se indican que las escalas utilizadas no han sido sometidas a unos procesos de traducción y retrotraducción garantizados según plantea las directrices de la Comisión Internacional de Tests (ITC) para la adaptación de los tests de unas culturas a otras. Por otra parte, y como ya se ha indicado con anterioridad, el reducido tamaño muestral limita el proceso de validación en lo que respecta a la medición la fiabilidad. El tamaño de la muestra es reducido debido a que la población a estudio (unas 20.000 personas aproximadamente, ya que en esa zona no hay un censo) y el tiempo de intervención (seis meses) fueron pequeños.

En pacientes en los que se observaba un daño tisular grave, mientras que con las dos primeras escalas su valoración era muy baja, con la escala Campbell se observaban muestras claras de dolor como gruñidos frecuentes y dificultad para confortar (ítems correspondientes a una puntuación alta en esta escala), lo cual hace dudar sobre si los pacientes realmente comprendían la relación de un número o una cara al dolor presente.

La muestra de este estudio resulta pequeña si lo que se pretende es en un futuro demostrar su efectividad en un contexto mayor, como la atención en catástrofes o ayuda humanitaria.
En resumen, las conclusiones se detallan en los siguientes puntos:

- Las escalas EVA y FPS muestran poca efectividad, se ha visto que el uso de estas escalas no conlleva a un tratamiento de elección.

- La escala Campbell parece ser la mejor escala adaptada a la población turkana.

- La escala Campbell se muestra eficaz en la elección del tratamiento correcto.

- No existe evidencia estadística de relación entre las escalas EVA y FPS con la escala Campbell.

- La escala de Campbell se muestra eficaz aun existiendo diferentes observadores.

- La valoración con escalas basadas en la asociación de un número o una cara muestra deficiencia, mientras que el resultado de un uso combinado de estas con una escala como la escala Campbell es más satisfactorio.

- Resulta necesaria la realización de más estudios para corroborar la validación de una escala como la Campbell o una modificación de la misma para poder extrapolarla a otros países con poblaciones con características similares en un contexto de urgencias.

\section{Agradecimientos}

A la población de Turkana, por su sencillez y autenticidad, y su total entrega y colaboración con el estudio. A Josephine, no solo nuestra "traductora local", sino también amiga, madre, psicóloga y una verdadera turkana. A la Misión Comunitaria San Pablo Apóstol, por su apoyo logístico y la aportación de todo tipo de facilidades posibles. A la Asociación de Enfermería Comunitaria (AEC), por su confianza y dedicación incondicional al desarrollo de la Enfermería.

\section{Financiación \\ Ninguna.}

\section{Conflicto de intereses}

Ninguno.

\section{$\overline{\text { Bibliografía }}$}

1. Lema.rae.es. Diccionario de la Lengua Española [Internet]. 2015 [citado 3 feb 2016]. Disponible en: http:// lema.rae.es/drae/?val=dolor

2. Abdalrahim MS, Majali SA, Stomberg MW, Bergbom I. The effect of postoperative pain management program on improving nurses' knowledge and attitudes toward pain. Nurs Educ Pract. 201 1; 11 (4): 250-5.

3. Ibarra E. Una nueva definición del dolor "Un imperativo de nuestros días". Rev Soc Esp Dolor 2006; 2:65-72.

4. Giordano J, Abramson K, Boswell MV. Pain Assessment: Subjectivity, Objectivity, and the Use of Neurotechnology. Part One: Practical and Ethical Issues. Pain Physician. 2010; 13:305-15.

5. Mossey JM. Defining racial and ethnic disparities in pain management. Clin Orthop Relat Resea. 2011; 469(7):85-90. 
6. Nortje N, Albertyn R. The cultural language of pain: a South African study. South Afr Fam Prac. 2015; 57(1):24-7.

7. Harding R, Powell RA, Kiyange F, Downing J, Mwangi-Powell F. Provision of pain- and symptom-relieving drugs for HIV/AIDS in sub-Saharan Africa. Pain Symp Manag. 2010; 40:405-15.

8. Huang K, Owino C, Vreeman RC, Hagembe M, Njuguna F, Strother RM, et al. Assessment of the face validity of two pain scales in Kenya: a validation study using cognitive interviewing. BMC Pall Care. 2012; $11: 5$.

9. Scholten WK, Milani B. Providing paedriatic palliative care in Kenya. Lancet. 2010; 376:846.

10. Pardo C, Muñoz T, Chamorro C. Monitorización del dolor. Recomendaciones del grupo de trabajo de analgesia y sedación de la SEMICYUC. Med Intens. 2008; 32(1):38-44.

11. Latorre Marco I, Solís Muñoz M, Falero Ruiz T. Grupo del proyecto de Investigación ESCID. Validación de la Escala de Conductas Indicadoras de Dolor para valorar el dolor en pacientes críticos, no comunicativos y sometidos a ventilación mecánica: resultados del proyecto ESCID. Enferm Intensiva. 201 1; 22:3, 12-26.

12. Camps T, Barbás C, Soto JM, García E, Camps J. Malalt amb dolor agut. En: Balliu A, Barbas C, Balmaseda A, et al. Guia d'Actuació Infermera d'Urgències I Emergències Prehospitalàries. Barcelona: Sistema d'Emergències Mèdiques; 2015. p. 179-84.

13. Clarett M, Plavotsky V. Escalas de evaluación del dolor y protocolo de analgesia en terapia intensiva. Instituto Argentino de Diagnóstico y Tratamiento; 2012.
14. Pérez Cruz I, Cepero Val ML, Santana Sardañas S, Expósito Palmero 0. Manejo del dolor en edad pediátrica. Artículo de revisión. Mediciego. 2013; 19 (supl. 2).

15. Covarrubias Gómez A. El manejo del dolor agudo postoperatorio: una década de experiencias. Rev Mex de Anest. 2013; 36(1):179-82.

16. Soyannwo OA, Amanor-Boadu SD, Sanya AO, Gureje O. Pain assessment in Nigerians -visual analogue scale and verbal rating scale compared. West Afr J Med. 2000; 19:242-5.

17. Blum D, Selman LE, Agupio G, Mashao T, Mmoledi K, Moll T, et al. Self-report measurement of pain \& symptoms in palliative care patients: a comparison of verbal, visual and hand scoring methods in Sub-Saharan Africa. Health Qualit Lif Outc. 2014; 12:1 18.

18. Huang KTL, Owino C, Gramelspacher GP, Monahan PO, Tabbey R, Hagembe M, et al. Prevalence and correlates of pain and pain treatment in a western Kenya referral hospital. J Palliat Med. 2013; 16(10):1260-7.

19. Bijur PE, Silver W, Gallagher E]. Reliability of the visual analog scale for measurement of acute pain. Acad Emerg Med. 2001; 8:1 153-7.

Si quieres ampliar más información sobre este artículo, consulta:

\begin{tabular}{|ll|}
\hline Introduce estos & \\
términos: & Dolor; diferencias culturales; escalas de \\
encuentrog & valoración; urgencias; Campbell. \\
\hline ENerembranteusente &
\end{tabular}

\section{Contacta con los autores en:}

egarciahigon@gmail.com 